Revised on October 3, 2003

\title{
Plane waves with negative phase velocity in Faraday chiral mediums
}

\author{
Tom G. Mackay* \\ School of Mathematics, University of Edinburgh \\ James Clerk Maxwell Building, The King's Buildings \\ Edinburgh EH9 3JZ, United Kingdom
}

Akhlesh Lakhtakia ${ }^{\dagger}$
CATMAS - Computational and Theoretical Materials Sciences Group
Department of Engineering Science and Mechanics
Pennsylvania State University, University Park, PA 16802-6812, USA

\begin{abstract}
The propagation of plane waves in a Faraday chiral medium is investigated. Conditions for the phase velocity to be directed opposite to the direction of power flow are derived for propagation in an arbitrary direction; simplified conditions which apply to propagation parallel to the distinguished axis are also established. These negative phase-velocity conditions are explored numerically using a representative Faraday chiral medium, arising from the homogenization of an isotropic chiral medium and a magnetically biased ferrite. It is demonstrated that the phase velocity may be directed opposite to power flow, provided that the gyrotropic parameter of the ferrite component medium is sufficiently large compared with the corresponding nongyrotropic permeability parameters.
\end{abstract}

PACS number(s): 41.20.Jb, 42.25.Bs, 83.80.Ab

\section{Introduction}

Homogeneous mediums which support the propagation of waves with phase velocity directed opposite to the direction of power flow have attracted much attention lately $[1,2,3]$. The archetypal example of such a medium is the isotropic dielectric-magnetic medium with simultaneously negative real permittivity and negative real permeability scalars, as first described Veselago in the late 1960s [4]. A range of exotic and potentially useful electromagnetic phenomenons, such as negative refraction, inverse Doppler shift, and inverse Čerenkov radiation, were predicted for this type of medium [3,4]. Recent experimental studies involving the microwave illumination of certain composite metamaterials $[5,6]$ — which followed on from earlier works of Pendry et al. $[7,8]$ — are supportive of Veselago's predictions and have prompted an intensification of interest in this area.

*Fax: +44 131650 6553; e-mail: T.Mackay@ed.ac.uk

${ }^{\dagger}$ Fax: +1 814863 7967; e-mail: axl4@psu.edu 
In particular, a general condition - applicable to dissipative isotropic dielectric-magnetic mediums - has been derived for the phase velocity to be directed opposite to power flow [9]; and this condition shows that the real parts of both the permittivity and the permeability scalars do not have to be negative.

A consensus has yet to be reached on terminology. For the present purposes, a medium supporting wave propagation with phase velocity directed opposite to power flow is most aptly referred to as a negative phase-velocity medium. However, the reader is alerted that alternative terms, such as left-handed material [3], backward medium [10], double-negative medium [1], and negative-index medium [11], are also in circulation. A discussion of this issue is available elsewhere [12].

The scope for the phase velocity to be directed opposite to power flow may be greatly extended by considering non-isotropic mediums, as has been indicated by considerations of uniaxial dielectric-magnetic mediums $[10,13,14]$. The focus of the present communication is the propagation of negative phase-velocity plane waves in Faraday chiral mediums (FCMs) [15, 16]. These mediums combine natural optical activity — as exhibited by isotropic chiral mediums [17] — with Faraday rotation - as exhibited by gyrotropic mediums [18, 19, 20]. A FCM may be theoretically conceptualized as a homogenized composite medium (HCM) arising from the blending together of an isotropic chiral medium with either a magnetically biased ferrite [21] or a magnetically biased plasma [22]. The HCM component mediums are envisioned as random particulate distributions. The homogenization process is justified provided that the particulate length scales in the mixture of components are small compared with electromagnetic wavelengths. A vast literature on the estimation of constitutive parameters of HCMs exists; see Refs. [23, 24], for example. The constitutive relations of FCMs have been rigorously established for some time [16], although inappropriate use still occurs [25].

In the following sections, wavenumbers and corresponding electric field phasors are delineated from eigenvalue/vector analysis for planewave propagation in an arbitrary direction. Simplified expressions for these quantities are derived for propagation parallel to the biasing (quasi)magnetostatic field [19, p. 71]. A general condition for the phase velocity to be directed opposite to power flow is established. The theoretical analysis is illustrated by means of a representative numerical example: the constitutive parameters of FCMs arising from a specific homogenization scenario are estimated and then used to explore the wave propagation characteristics.

As regards notation, vectors are underlined whereas dyadics are double underlined. All electromagnetic field phasors and constitutive parameters depend implicitly on the circular frequency $\omega$ of the electromagnetic field. Unit vectors are denoted by the superscript ${ }^{\wedge}$ symbol, while $\underline{\underline{I}}=\underline{\hat{x}} \underline{\hat{x}}+\underline{\hat{y}} \hat{\hat{y}}+\underline{\hat{z}} \underline{\hat{z}}$ is the identity dyadic. The complex conjugate of a quantity $q$ is written as $\bar{q}^{*}$; the real part of $q$ is written as $\operatorname{Re}\{q\}$. The free-space (i.e., vacuum) wavenumber is denoted by $k_{0}=\omega \sqrt{\epsilon_{0} \mu_{0}}$ where $\epsilon_{0}$ and $\mu_{0}$ are the permittivity and permeability of free space, respectively; and $\eta_{0}=\sqrt{\mu_{0} / \epsilon_{0}}$ represents the intrinsic impedance of free space. 


\section{Analysis}

\subsection{Preliminaries}

The propagation of plane waves with field phasors

$$
\left.\begin{array}{l}
\underline{E}(\underline{r})=\underline{E}_{0} \exp \left(i k_{0} \tilde{k} \underline{\hat{u}} \cdot \underline{r}\right) \\
\underline{H}(\underline{r})=\underline{H}_{0} \exp \left(i k_{0} \tilde{k} \underline{\hat{u}} \cdot \underline{r}\right)
\end{array}\right\}
$$

in a FCM is considered. Such a medium is characterized by the frequency-domain constitutive relations [16]

$$
\left.\begin{array}{l}
\underline{D}(\underline{r})=\underline{\underline{\epsilon}} \cdot \underline{E}(\underline{r})+\underline{\underline{\xi}} \cdot \underline{H}(\underline{r}) \\
\underline{B}(\underline{r})=-\underline{\underline{\xi}} \cdot \underline{E}(\underline{r})+\underline{\underline{\mu}} \cdot \underline{H}(\underline{r})
\end{array}\right\},
$$

with constitutive dyadics

$$
\left.\begin{array}{l}
\underline{\underline{\epsilon}}=\epsilon_{0}\left[\epsilon \underline{\underline{I}}-i \epsilon_{g} \underline{\hat{z}} \times \underline{\underline{I}}+\left(\epsilon_{z}-\epsilon\right) \underline{\hat{z}} \underline{\hat{z}}\right] \\
\underline{\underline{\xi}}=i \sqrt{\epsilon_{0} \mu_{0}}\left[\xi \underline{\underline{I}}-i \xi_{g} \underline{\hat{z}} \times \underline{\underline{I}}+\left(\xi_{z}-\xi\right) \underline{\hat{z}} \hat{\underline{z}}\right] \\
\underline{\underline{\mu}}=\mu_{0}\left[\mu \underline{\underline{I}}-i \mu_{g} \underline{\hat{z}} \times \underline{\underline{I}}+\left(\mu_{z}-\mu\right) \underline{\hat{z}} \underline{\hat{z}}\right]
\end{array}\right\} .
$$

Thus, the distinguished axis of the FCM is chosen to be the $z$ axis. For FCMs which arise as HCMs, the gyrotropic parameters $\epsilon_{g}, \xi_{g}$ and $\mu_{g}$ in (3) develop due to the gyrotropic properties of the ferrite or plasma component mediums. Parenthetically, it is remarked that more general FCMs can develop through the homogenization of component mediums based on nonspherical particulate geometries $[22,26]$.

In general, the relative wavenumber $\tilde{k}$ in (1) is complex valued; i.e.,

$$
\tilde{k}=\tilde{k}_{R}+i \tilde{k}_{I}, \quad\left(\tilde{k}_{R}, \tilde{k}_{I} \in \mathbb{R}\right) .
$$

It may be calculated from the planewave dispersion relation

$$
\operatorname{det}\left[\underline{\underline{L}}\left(i k_{0} \tilde{k} \underline{\hat{u}}\right)\right]=0
$$

which arises from the vector Helmholtz equation

$$
\underline{\underline{L}}(\nabla) \cdot \underline{E}(\underline{r})=\underline{0}
$$

wherein

$$
\underline{\underline{L}}(\nabla)=(\nabla \times \underline{\underline{I}}+i \omega \underline{\underline{\xi}}) \cdot \underline{\underline{\mu}}^{-1} \cdot(\nabla \times \underline{\underline{I}}+i \omega \underline{\underline{\xi}})-\omega^{2} \underline{\underline{\epsilon}}
$$

Of particular interest is the orientation of the phase velocity, as specified by the direction of $\tilde{k}_{R} \underline{\hat{u}}$, relative to the direction of power flow given by the time-averaged Poynting vector $\underline{P}(\underline{r})=$ 
$\frac{1}{2} \operatorname{Re}\left[\underline{E}(\underline{r}) \times \underline{H}^{*}(\underline{r})\right]$. The combination of the constitutive relations (2) with the source-free Maxwell curl postulates

$$
\left.\begin{array}{l}
\nabla \times \underline{E}(\underline{r})=i \omega \underline{B}(\underline{r}) \\
\nabla \times \underline{H}(\underline{r})=-i \omega \underline{D}(\underline{r})
\end{array}\right\}
$$

yields

$$
\underline{P}(\underline{r})=\frac{1}{2} \exp \left(-2 k_{0} \tilde{k}_{I} \underline{\hat{u}} \cdot \underline{r}\right) \operatorname{Re}\left\{\underline{E}_{0} \times\left[\left(\underline{\mu}^{-1}\right)^{*} \cdot\left(\sqrt{\epsilon_{0} \mu_{0}} \tilde{k}^{*} \underline{\hat{u}} \times \underline{E}_{0}^{*}+\underline{\xi}^{*} \cdot \underline{E}_{0}^{*}\right)\right]\right\}
$$

for plane waves (1).

In the remainder of this section, the quantity $\tilde{k}_{R} \underline{\hat{u}} \cdot \underline{P}(\underline{r})$ is derived for planewave propagation in an arbitrary direction; without loss of generality, $\underline{\hat{u}}$ is taken to lie in the $x z$ plane (i.e., $\underline{\hat{u}}=$ $\underline{\hat{x}} \sin \theta+\underline{\hat{z}} \cos \theta)$. Further manipulations reveal the simple form $\tilde{k}_{R} \underline{\hat{u}} \cdot \underline{P}(\underline{r})$ adopts for propagation along the FCM distinguished axis (i.e., $\underline{\hat{u}}=\underline{\hat{z}}$ ).

\subsection{Propagation in the $x z$ plane}

For $\underline{\hat{u}}=\underline{\hat{x}} \sin \theta+\underline{\hat{z}} \cos \theta$, the dispersion relation (5) may be represented by the quartic polynomial

$$
a_{4} \tilde{k}^{4}+a_{3} \tilde{k}^{3}+a_{2} \tilde{k}^{2}+a_{1} \tilde{k}+a_{0}=0
$$

with coefficients

$$
\begin{aligned}
a_{4}= & \left(\epsilon \sin ^{2} \theta+\epsilon_{z} \cos ^{2} \theta\right)\left(\mu \sin ^{2} \theta+\mu_{z} \cos ^{2} \theta\right)-\left(\xi \sin ^{2} \theta+\xi_{z} \cos ^{2} \theta\right)^{2}, \\
a_{3}= & 2 \cos \theta\left\{\sin ^{2} \theta\left[\mu_{g}\left(\epsilon \xi_{z}-\epsilon_{z} \xi\right)+\epsilon_{g}\left(\mu \xi_{z}-\mu_{z} \xi\right)+\xi_{g}\left(\mu \epsilon_{z}+\epsilon \mu_{z}-2 \xi \xi_{g}\right)\right]\right. \\
& \left.+2 \cos ^{2} \theta \xi_{g}\left(\epsilon_{z} \mu_{z}-\xi_{z}^{2}\right)\right\} \\
a_{2}= & \sin ^{2} \theta\left\{\mu \mu_{z}\left(\epsilon_{g}^{2}-\epsilon^{2}\right)+\left(\xi^{2}+\xi_{g}^{2}\right)\left(\mu \epsilon_{z}+\epsilon \mu_{z}\right)-2 \xi\left[\xi_{z}\left(\xi_{g}^{2}-\xi^{2}\right)+\mu_{g} \epsilon_{z} \xi_{g}\right]\right. \\
& \left.-2 \epsilon_{g}\left[\xi_{z}\left(\mu_{g} \xi-\mu \xi_{g}\right)+\mu_{z} \xi \xi_{g}\right]-\epsilon\left[\epsilon_{z}\left(\mu^{2}-\mu_{g}^{2}\right)+2 \xi_{z}\left(\mu \xi-\mu_{g} \xi_{g}\right)\right]\right\} \\
& +2 \cos ^{2} \theta\left(\epsilon_{z} \mu_{z}-\xi_{z}^{2}\right)\left(3 \xi_{g}^{2}-\xi^{2}-\epsilon_{g} \mu_{g}-\epsilon \mu\right), \\
a_{1}= & 4 \cos \theta\left(\epsilon_{z} \mu_{z}-\xi_{z}^{2}\right)\left[\xi\left(\epsilon_{g} \mu+\epsilon \mu_{g}\right)+\xi_{g}\left(\xi_{g}^{2}-\xi^{2}-\epsilon \mu-\epsilon_{g} \mu_{g}\right)\right], \\
a_{0}= & \left(\epsilon_{z} \mu_{z}-\xi_{z}^{2}\right)\left[\left(\epsilon^{2}-\epsilon_{g}^{2}\right)\left(\mu^{2}-\mu_{g}^{2}\right)+\left(\xi_{g}^{2}-\xi^{2}\right)^{2}-2\left(\xi_{g}^{2}+\xi^{2}\right)\left(\epsilon \mu+\epsilon_{g} \mu_{g}\right)\right. \\
& \left.+4 \xi \xi_{g}\left(\epsilon \mu_{g}+\mu \epsilon_{g}\right)\right] .
\end{aligned}
$$

Hence, four relative wavenumbers $\tilde{k}=\kappa_{i}, \kappa_{i i}, \kappa_{i i i}$ and $\kappa_{i v}$ may be extracted - either algebraically or numerically [27] — as the roots of (10).

Upon substituting $\underline{\hat{u}}=\underline{\hat{\hat{x}}} \sin \theta+\underline{\hat{z}} \cos \theta$ into (9) and combining with (3), the component of $\underline{P}(\underline{r})$ 
aligned with $\underline{\hat{u}}$ emerges straightforwardly as

$$
\begin{aligned}
\underline{\hat{u}} \cdot \underline{P}(\underline{r})= & \frac{1}{2 \eta_{0}} \exp \left(-2 k_{0} \tilde{k}_{I} \underline{\hat{u}} \cdot \underline{r}\right) \operatorname{Re}\left\{\frac{1}{\mu_{z}^{*}}\left(\tilde{k}^{*} \sin \theta\left|E_{0 y}\right|^{2}-i \xi_{z}^{*} E_{0 y} E_{0 z}^{*}\right) \sin \theta\right. \\
& +\frac{1}{\left(\mu^{*}\right)^{2}-\left(\mu_{g}^{*}\right)^{2}}\left[\tilde { k } ^ { * } \left(\left[\mu^{*}\left(\left|E_{0 x}\right|^{2}+\left|E_{0 y}\right|^{2}\right)+i \mu_{g}^{*}\left(E_{0 x} E_{0 y}^{*}-E_{0 y} E_{0 x}^{*}\right)\right] \cos ^{2} \theta\right.\right. \\
& \left.+\mu^{*}\left|E_{0 z}\right|^{2} \sin ^{2} \theta-\left[\mu^{*}\left(E_{0 z} E_{0 x}^{*}+E_{0 x} E_{0 z}^{*}\right)+i \mu_{g}^{*}\left(E_{0 z} E_{0 y}^{*}-E_{0 y} E_{0 z}^{*}\right)\right] \sin \theta \cos \theta\right) \\
& +\left(\mu^{*} \xi_{g}^{*}-\mu_{g}^{*} \xi^{*}\right)\left[\left(\left|E_{0 x}\right|^{2}+\left|E_{0 y}\right|^{2}\right) \cos \theta-E_{0 z} E_{0 x}^{*} \sin \theta\right] \\
& \left.\left.-i\left(\mu^{*} \xi^{*}-\mu_{g}^{*} \xi_{g}^{*}\right)\left[\left(E_{0 x} E_{0 y}^{*}-E_{0 x}^{*} E_{0 y}\right) \cos \theta-E_{0 z} E_{0 y}^{*} \sin \theta\right]\right]\right\},
\end{aligned}
$$

wherein $\left(E_{0 x}, E_{0 y}, E_{0 z}\right)=\underline{E}_{0}$.

Let the quantity

$$
w=2 \eta_{0} \exp \left(2 k_{0} \tilde{k}_{I} \underline{\hat{u}} \cdot \underline{r}\right)\left|E_{0 y}\right|^{-2} \tilde{k}_{R} \underline{\hat{u}} \cdot \underline{P}(\underline{r})
$$

be introduced such that the fulfilment of the negative phase-velocity condition

$$
\tilde{k}_{R} \underline{\hat{u}} \cdot \underline{P}(\underline{r})<0
$$

is signaled by $w<0$.

Substitution of (16) in (17) yields the expression

$$
\begin{aligned}
w= & \tilde{k}_{R} \operatorname{Re}\left\{\frac{1}{\mu_{z}^{*}}\left(\tilde{k}^{*} \sin \theta-i \xi_{z}^{*} \beta^{*}\right) \sin \theta\right. \\
& +\frac{1}{\left(\mu^{*}\right)^{2}-\left(\mu_{g}^{*}\right)^{2}}\left[\tilde { k } ^ { * } \left(\left[\mu^{*}\left(|\alpha|^{2}+1\right)+i \mu_{g}^{*}\left(\alpha-\alpha^{*}\right)\right] \cos ^{2} \theta\right.\right. \\
& \left.+\mu^{*}|\beta|^{2} \sin ^{2} \theta-\left[\mu^{*}\left(\alpha^{*} \beta+\alpha \beta^{*}\right)+i \mu_{g}^{*}\left(\beta-\beta^{*}\right)\right] \sin \theta \cos \theta\right) \\
& +\left(\mu^{*} \xi_{g}^{*}-\mu_{g}^{*} \xi^{*}\right)\left[\left(|\alpha|^{2}+1\right) \cos \theta-\alpha^{*} \beta \sin \theta\right] \\
& \left.-i\left(\mu^{*} \xi^{*}-\mu_{g}^{*} \xi_{g}^{*}\right)\left[\left(\alpha-\alpha^{*}\right) \cos \theta-\beta \sin \theta\right]\right\} .
\end{aligned}
$$

The ratios of electric field components

$$
\left.\begin{array}{l}
\alpha=E_{0 x} / E_{0 y} \\
\beta=E_{0 z} / E_{0 y}
\end{array}\right\}
$$

in (19) are derived as follows: As a function of $\theta$, the dyadic operator $\underline{\underline{L}}$ of $(7)$ has the form

$$
\begin{gathered}
\underline{\underline{L}=}=i k_{0}\left\{[\underline{\underline{L}}]_{11} \underline{\hat{x}} \underline{\hat{x}}+[\underline{\underline{L}}]_{22} \underline{\hat{y}} \underline{\hat{y}}+[\underline{\underline{L}}]_{33} \underline{\hat{z}} \underline{\hat{z}}+[\underline{\underline{L}}]_{12}(\underline{\hat{x}} \underline{\hat{y}}-\underline{\hat{y}} \underline{\hat{x}})\right. \\
\left.+[\underline{\underline{L}}]_{13}(\underline{\hat{x}} \underline{\hat{z}}+\underline{\hat{z}} \underline{\hat{x}})+[\underline{\underline{L}}]_{23}(\underline{\hat{y}} \underline{\hat{z}}-\underline{\hat{z}} \underline{\hat{y}})\right\},
\end{gathered}
$$


with components

$$
\begin{aligned}
& {[\underline{\underline{L}}]_{11}=\epsilon+\frac{2 \mu_{g} \xi \Gamma-\mu\left(\xi^{2}+\Gamma^{2}\right)}{\mu^{2}-\mu_{g}^{2}}} \\
& {[\underline{\underline{L}}]_{22}=\epsilon-\frac{\tilde{k}^{2} \sin ^{2} \theta}{\mu_{z}}+\frac{2 \mu_{g} \xi \Gamma-\mu\left(\xi^{2}+\Gamma^{2}\right)}{\mu^{2}-\mu_{g}^{2}}} \\
& {[\underline{\underline{L}}]_{33}=\epsilon_{z}-\frac{\xi_{z}^{2}}{\mu_{z}}-\frac{\mu \tilde{k}^{2} \sin ^{2} \theta}{\mu^{2}-\mu_{g}^{2}}} \\
& {[\underline{\underline{L}}]_{12}=i\left(\epsilon_{g}+\frac{\mu_{g}\left(\xi^{2}+\Gamma^{2}\right)-2 \mu \xi \Gamma}{\mu^{2}-\mu_{g}^{2}}\right)} \\
& {[\underline{\underline{L}}]_{13}=\frac{\mu \Gamma-\mu_{g} \xi}{\mu^{2}-\mu_{g}^{2}} \tilde{k} \sin \theta} \\
& {[\underline{\underline{L}}]_{23}=i\left(\frac{\mu_{g} \Gamma-\mu \xi}{\mu^{2}-\mu_{g}^{2}}-\frac{\xi_{z}}{\mu_{z}}\right) \tilde{k} \sin \theta}
\end{aligned}
$$

where $\Gamma=\xi_{g}+\tilde{k} \cos \theta$. It follows from the vector Helmholtz equation (6) that

$$
\left.\begin{array}{rl}
\alpha & =\frac{[\underline{\underline{\underline{L}}}]_{12}[\underline{\underline{L}}]_{33}+[\underline{\underline{\underline{L}}}]_{13}[\underline{\underline{\underline{L}}}]_{23}}{[\underline{\underline{\underline{L}}}]_{13}[\underline{\underline{L}}]_{13}-[\underline{\underline{L}}]_{11}[\underline{\underline{\underline{L}}}]_{33}} \\
\beta & =\frac{[\underline{\underline{\underline{L}}}]_{12}[\underline{\underline{\underline{L}}}]_{23}-[\underline{\underline{\underline{L}}}]_{13}[\underline{\underline{\underline{L}}}]_{22}}{[\underline{\underline{\underline{L}}}]_{13}[\underline{\underline{L}}]_{23}+[\underline{\underline{\underline{L}}}]_{12}[\underline{\underline{\underline{L}}}]_{33}}
\end{array}\right\} .
$$

\subsection{Propagation along the $z$ axis}

The results of the preceding analysis simplify considerably for planewave propagation along the $z$ axis (i.e., $\theta=0$ ). The quartic dispersion relation (10) yields the four relative wavenumbers

$$
\left.\begin{array}{l}
\kappa_{i}=\sqrt{\epsilon+\epsilon_{g}} \sqrt{\mu+\mu_{g}}-\xi-\xi_{g} \\
\kappa_{i i}=-\sqrt{\epsilon+\epsilon_{g}} \sqrt{\mu+\mu_{g}}-\xi-\xi \\
\kappa_{i i i}=\sqrt{\epsilon-\epsilon_{g}} \sqrt{\mu-\mu_{g}}+\xi-\xi_{g} \\
\kappa_{i v}=-\sqrt{\epsilon-\epsilon_{g}} \sqrt{\mu-\mu_{g}}+\xi-\xi_{g}
\end{array}\right\}
$$

and (19) reduces to

$$
w=\tilde{k}_{R} \operatorname{Re}\left\{\frac{\left(|\alpha|^{2}+1\right)\left(\tilde{k}^{*} \mu^{*}-\mu_{g}^{*} \xi^{*}+\mu^{*} \xi_{g}^{*}\right)+i\left(\alpha-\alpha^{*}\right)\left(\tilde{k}^{*} \mu_{g}^{*}-\mu^{*} \xi^{*}+\mu_{g}^{*} \xi_{g}^{*}\right)}{\left(\mu^{*}\right)^{2}-\left(\mu_{g}^{*}\right)^{2}}\right\} .
$$

Since the dyadic operator components $[\underline{\underline{L}}]_{13}$ and $[\underline{\underline{L}}]_{23}$ are null-valued for $\theta=0$, the electric field ratios are given as

$$
\left.\begin{array}{l}
\alpha=-[\underline{\underline{L}}]_{12} /[\underline{\underline{L}}]_{11} \\
\beta=0
\end{array}\right\}
$$

Note that a further consequence of $[\underline{\underline{L}}]_{13}=[\underline{\underline{L}}]_{23}=0$ is that the time-averaged Poynting vector is parallel to the $z$ axis. 
By substituting (29) into (22) and (25), the ratio $\alpha$ emerges as

$$
\alpha=\left\{\begin{array}{lll}
i & \text { for } & \tilde{k}=\kappa_{i}, \kappa_{i i}, \\
-i & \text { for } & \tilde{k}=\kappa_{i i i}, \kappa_{i v} .
\end{array}\right.
$$

Hence, negative-phase velocity propagation along the $z$ axis occurs provided $w<0$ where

$$
\begin{aligned}
& w=w_{i}=2 \operatorname{Re}\left\{\sqrt{\epsilon+\epsilon_{g}} \sqrt{\mu+\mu_{g}}-\xi-\xi_{g}\right\} \operatorname{Re}\left\{\frac{\sqrt{\epsilon^{*}+\epsilon_{g}^{*}}}{\sqrt{\mu^{*}+\mu_{g}^{*}}}\right\} \quad \text { for } \quad \tilde{k}=\kappa_{i}, \\
& w=w_{i i}=2 \operatorname{Re}\left\{\sqrt{\epsilon+\epsilon_{g}} \sqrt{\mu+\mu_{g}}+\xi+\xi_{g}\right\} \operatorname{Re}\left\{\frac{\sqrt{\epsilon^{*}+\epsilon_{g}^{*}}}{\sqrt{\mu^{*}+\mu_{g}^{*}}}\right\} \quad \text { for } \quad \tilde{k}=\kappa_{i i}, \\
& w=w_{i i i}=2 \operatorname{Re}\left\{\sqrt{\epsilon-\epsilon_{g}} \sqrt{\mu-\mu_{g}}+\xi-\xi_{g}\right\} \operatorname{Re}\left\{\frac{\sqrt{\epsilon^{*}-\epsilon_{g}^{*}}}{\sqrt{\mu^{*}-\mu_{g}^{*}}}\right\} \quad \text { for } \quad \tilde{k}=\kappa_{i i i}, \\
& w=w_{i v}=2 \operatorname{Re}\left\{\sqrt{\epsilon-\epsilon_{g}} \sqrt{\mu-\mu_{g}}-\xi+\xi_{g}\right\} \operatorname{Re}\left\{\frac{\sqrt{\epsilon^{*}-\epsilon_{g}^{*}}}{\sqrt{\mu^{*}-\mu_{g}^{*}}}\right\} \quad \text { for } \quad \tilde{k}=\kappa_{i v} .
\end{aligned}
$$

\section{Numerical results}

In order to further examine the negative phase-velocity conditions derived in Sections 2.2 and 2.3, let us consider a Faraday chiral medium (FCM) produced by mixing (a) an isotropic chiral medium described by the constitutive relations [17]

$$
\left.\begin{array}{l}
\underline{D}=\epsilon_{0} \epsilon^{a} \underline{E}+i \sqrt{\epsilon_{0} \mu_{0}} \xi^{a} \underline{H} \\
\underline{B}=-i \sqrt{\epsilon_{0} \mu_{0}} \xi^{a} \underline{E}+\mu_{0} \mu^{a} \underline{H}
\end{array}\right\}
$$

and (b) a magnetically biased ferrite described by the constitutive relations $[19$, Ch. 7$]$

$$
\left.\begin{array}{l}
\underline{D}=\epsilon_{0} \epsilon^{b} \underline{E} \\
\underline{B}=\mu_{0}\left[\mu^{b} \underline{\underline{I}}-i \mu_{g}^{b} \underline{\underline{z}} \times \underline{\underline{I}}+\left(\mu_{z}^{b}-\mu^{b}\right) \underline{\hat{z}} \underline{\hat{z}}\right] \cdot \underline{H}
\end{array}\right\} .
$$

Both component mediums are envisioned as random distributions of electrically small, spherical particles. The resulting homogenized composite medium (HCM) is a FCM characterized by the constitutive dyadics

$$
\left.\begin{array}{l}
\underline{\underline{\epsilon}}^{H C M}=\epsilon_{0}\left[\epsilon^{H C M} \underline{\underline{I}}-i \epsilon_{g}^{H C M} \underline{\underline{z}} \times \underline{\underline{I}}+\left(\epsilon_{z}^{H C M}-\epsilon^{H C M}\right) \underline{\hat{z}} \underline{\hat{z}}\right] \\
\underline{\underline{\xi}}^{H C M}=i \sqrt{\epsilon_{0} \mu_{0}}\left[\xi^{H C M} \underline{\underline{I}}-i \xi_{g}^{H C M} \underline{\hat{z}} \times \underline{\underline{I}}+\left(\xi_{z}^{H C M}-\xi^{H C M}\right) \underline{\hat{z}} \hat{\underline{z}}\right] \\
\underline{\underline{\mu}}^{H C M}=\mu_{0}\left[\mu^{H C M} \underline{\underline{I}}-i \mu_{g}^{H C M} \underline{\hat{z}} \times \underline{\underline{I}}+\left(\mu_{z}^{H C M}-\mu^{H C M}\right) \underline{\hat{z}} \underline{\hat{z}}\right]
\end{array}\right\} .
$$


Incidentally, a FCM with constitutive dyadics of the form (39) may also be developed via the homogenization of an isotropic chiral medium and a magnetically biased plasma [22].

The constitutive dyadics $\underline{\underline{\epsilon}}^{H C M}, \underline{\underline{\xi}}^{H C M}$ and $\underline{\underline{\mu}}^{H C M}$ are estimated using the Bruggeman homogenization formalism for a representative example. Comprehensive details of the Bruggeman formalism $[28,24]$ and its implementation in the context of FCMs $[21,22,29]$ are available elsewhere. Initially, we restrict our attention to nondissipative FCMs; the influence of dissipation is considered later in this section.

\subsection{Nondissipative FCMs}

The parameter values selected for nondissipative component mediums are as follows:

$$
\epsilon^{a}=3.2, \quad \xi^{a}=2.4, \quad \mu^{a}=2 ; \epsilon^{b}=2.2, \quad \mu^{b}=3.5, \quad \mu_{z}^{b}=1, \quad \mu_{g}^{b} \in[0,4] .
$$

The permeability parameters for component medium $b$ may be viewed in terms of the semi-classical ferrite model as

$$
\left.\begin{array}{rl}
\mu^{b} & =1+\frac{\omega_{0} \omega_{m}}{\omega_{0}^{2}-\omega^{2}} \\
\mu_{g}^{b} & =\frac{\omega \omega_{m}}{\omega_{0}^{2}-\omega^{2}} \\
\mu_{z}^{b} & =1
\end{array}\right\}
$$

wherein $\omega_{0}$ is the Larmor precessional frequency of spinning electrons and $\omega_{m}$ is the saturated magnetization frequency $[18,19]$. Thus, the parameter values $\mu^{b}=3.5$ and $\mu_{g}^{b} \in[0,4]$ correspond to the relative frequency range $\left(\omega_{0} / \omega\right) \in[0.625, \infty)$.

Let $f_{a}$ denote the volume fraction of the isotropic chiral component medium $a$. In figure 1 , the estimated constitutive parameters of the HCM are plotted as functions of $f_{a}$ for $\mu_{g}^{b}=4$. The uniaxial and gyrotropic characteristics of a FCM are clearly reflected by the constituents of the permeability dyadic $\underline{\mu}^{H C M}$ and the magnetoelectric dyadic $\underline{\xi}^{H C M}$. In contrast, the HCM is close to being isotropic with respect to its dielectric properties. $\overline{\bar{S}}$ ignificantly, eight of the nine scalars appearing in (39) are positive, while $\epsilon_{g}^{H C M}$ is negative only for $f_{a}<0.32$; however, $\left|\epsilon_{g}^{H C M}\right|<<$ $\left|\epsilon^{H C M}\right|$ and $\left|\epsilon_{g}^{H C M}\right|<<\left|\epsilon_{z}^{H C M}\right|$ for all values of $f_{a} \in[0,1]$.

The permeability parameters $\mu^{H C M}$ and $\mu_{g}^{H C M}$ are equal at $f_{a} \approx 0.25$, it being clear from the right side of (19) that this equality has an important bearing on the stability of $w$. Further calculations with $\mu_{g}^{b}=2$ and $\mu_{g}^{b}=3$ have confirmed that $\mu^{H C M} \neq \mu_{g}^{H C M}$ for all volume fractions $f_{a} \in[0,1]$. This matter is pursued in figure 2 where the estimated constitutive parameters of the HCM are graphed as functions of $\mu_{g}^{b}$ for $f_{a}=0.35$. The HCM gyrotropic parameters $\xi_{g}^{H C M}$ and $\mu_{g}^{H C M}$ are observed to increase steadily as $\mu_{g}^{b}$ increases; $\epsilon_{g}^{H C M}, \xi_{g}^{H C M}$ and $\mu_{g}^{H C M}$ all vanish in the limit $\mu_{g}^{b} \rightarrow 0$. Also, as $\mu_{g}^{b}$ increases, the degree of uniaxiality (with respect to the $z$ axis) increases for $\underline{\underline{\xi}}^{H C M}$ but decreases for $\underline{\underline{\mu}}^{H C M}$.

The relative wavenumbers $\tilde{k}=\kappa_{i-i v}$ for propagation along the $z$ axis, as specified in (29), are displayed in figure 3 as functions of $f_{a}$, for $\mu_{g}^{b}=2,3$ and 4 . The relative wavenumbers $\kappa_{i}>0$ and $\kappa_{i i}<0$ for all $f_{a} \in[0,1]$ for $\mu_{g}^{b}=2,3$ and 4 . Similarly, for $\mu_{g}^{b}=2$ and 3 , the relative numbers $\kappa_{i i i}>0$ and $\kappa_{i v}<0$. 
However, the equality $\mu^{H C M}=\mu_{g}^{H C M}$, which occurs at $f_{a} \approx 0.25$ for $\mu_{g}^{b}=4$, results in $\kappa_{i i i}$ and $\kappa_{i v}$ acquiring nonzero imaginary parts as $f_{a}$ falls below 0.25 for $\mu_{g}^{b}=4$. Only the real parts of these complex-valued relative wavenumbers are plotted in figure 3.

Observe that $\kappa_{i}, \kappa_{i i i}$ and $\kappa_{i v}>0$ in figure 3 , whereas $\kappa_{i i}<0$ in the volume fraction range $0.25<f_{a}<0.42$ with $\mu_{g}^{b}=4$. Furthermore, $\kappa_{i}$, $\operatorname{Re}\left\{\kappa_{i i i}\right\}$ and $\operatorname{Re}\left\{\kappa_{i v}\right\}>0$ while $\kappa_{i i}<0$ for $f_{a}<0.25$ with $\mu_{g}^{b}=4$. In the limit $f_{a} \rightarrow 0$, the relative wavenumbers $\kappa_{i-i v} \rightarrow \pm \sqrt{\epsilon^{b}} \sqrt{\mu^{b} \pm \mu_{g}^{b}}$ (i.e., the relative wavenumbers of a ferrite biased along the $z$ axis [19]). Also, as $f_{a} \rightarrow 1$, the relative wavenumbers $\kappa_{i-i v} \rightarrow \pm \sqrt{\epsilon^{a} \mu^{a}} \pm \xi^{a}$ (i.e., the relative wavenumbers of an isotropic chiral medium).

The values of $w$ corresponding to the relative wavenumbers $\kappa_{i-i v}$ of figure 3 , namely $w_{i-i v}$, are plotted against $f_{a}$ in figure 4 for $\mu_{g}^{b}=2,3$ and 4 . The quantities $w_{i-i i i} \geq 0$ for all volume fractions $f_{a} \in[0,1]$ with $\mu_{g}^{b}=2,3$ and 4 . Thus, for the relative wavenumbers $\kappa_{i-i i i}$, power flows in the same direction as the phase velocity. This is the case regardless of whether the phase velocity is directed along the positive $z$ axis (as in modes $\kappa_{i}$ and $\kappa_{i i i}$ ) or directed along the negative $z$ axis (as in mode $\kappa_{i i}$ ). Both $w_{i i i}$ and $w_{i v}$ are null valued in those regions where the corresponding relative wavenumbers, $\kappa_{i i i}$ and $\kappa_{i v}$, respectively, have nonzero imaginary parts. In addition, $w_{i i i} \rightarrow \infty$ and $w_{i v} \rightarrow-\infty$ in the vicinity of $f_{a}=0.25$ for $\mu_{g}^{b}=4$.

Significantly, $w_{i v}<0$ for $\mu_{g}^{b}=4$ at volume fractions $f_{a} \in(0.25,0.42)$ in figure 4 . This means that the negative phase-velocity condition then holds in the chosen FCM which has been conceptualized as a homogenized composite medium.

In figure 5 , the relative wavenumbers $\kappa_{i}$ and $\kappa_{i i i}$ for $\theta=\pi / 2$ (i.e., propagation along the $x$ axis) are plotted against $f_{a}$ for $\mu_{g}^{b}=2,3$ and 4 . The graphs of $\kappa_{i i}$ and $\kappa_{i v}$ need not be presented since $\kappa_{i}=-\kappa_{i i}$ and $\kappa_{i i i}=-\kappa_{i v} .{ }^{1}$ For all $f_{a} \in[0,1]$ with $\mu_{g}^{b}=2$ and 3 , the relative wavenumbers $\kappa_{i}>0$ and $\kappa_{i i i}<0$. Similarly, $\kappa_{i}>0$ for $\mu_{g}^{b}=4$. However, when $\mu_{g}^{b}=4$, it is found that $\kappa_{i i i}<0$ for $f_{a}>0.42$ but $\kappa_{i i i}$ possesses a nonzero imaginary part and $\operatorname{Re}\left\{\kappa_{i i i}\right\}=0$ for $f_{a}<0.42$. In the limit $f_{a} \rightarrow 0$, the relative wavenumbers $\kappa_{i-i v} \rightarrow \pm \sqrt{\epsilon^{b} / \mu^{b}} \sqrt{\left(\mu^{b}\right)^{2}-\left(\mu_{g}^{b}\right)^{2}}$ and $\pm \sqrt{\epsilon^{b} \mu_{z}^{b}}$ (i.e., the relative wavenumbers of a ferrite biased along the $x$ axis [19]). Also, as $f_{a} \rightarrow 1$, the relative wavenumbers $\kappa_{i-i v} \rightarrow \pm \sqrt{\epsilon^{a} \mu^{a}} \pm \xi^{a}$ (i.e., the relative wavenumbers of an isotropic chiral medium).

Figure 6 shows the plots of $w_{i, i i i}$ corresponding to the relative wavenumbers $\kappa_{i, i i i}$ of figure 5 . The graphs of $w=w_{i i, i v}$ corresponding to the relative waveumbers $\kappa_{i i, i v}$ are not displayed since the equalities $w_{i}=w_{i i}$ and $w_{i i i}=w_{i v}$ hold for $\theta=\pi / 2$ - as may be inferred from (19)-(28). The quantities $w_{i, i i i} \geq 0$ at all volume fractions $f_{a} \in[0,1]$ with $\mu_{g}^{b}=2,3$ and 4 . As remarked earlier for $\kappa_{i-i i i}$ propagation along the $z$ axis, here we have that power flows in the same direction as the phase velocity, regardless of whether the phase velocity is directed along the positive $x$ axis (mode $\kappa_{i}$ ) or along the negative $x$ axis (mode $\kappa_{i i i}$ ). Furthermore, it is found that $w_{i i i}=0$ in the region where the corresponding relative wavenumber $\kappa_{i i i}$ is purely imaginary (i.e., for $f_{a}<0.42$ with $\mu_{g}^{b}=4$ ).

\footnotetext{
${ }^{1}$ When $\theta=\pi / 2$, the dispersion relation (10) reduces to a quadratic polynomial in $\tilde{k}^{2}$.
} 


\subsection{Dissipative FCMs}

The scope of these numerical investigations is now broadened by considering (i) the effects of dissipation or loss; and (ii) propagation in an arbitrary direction. Let a small amount of loss be incorporated into component medium $b$ by selecting the constitutive parameters of the component mediums as

$$
\epsilon^{a}=3.2, \quad \xi^{a}=2.4, \quad \mu^{a}=2 ; \epsilon^{b}=2.2+i \delta, \quad \mu^{b}=3.5+i \delta, \quad \mu_{z}^{b}=1+i 0.5 \delta, \quad \mu_{g}^{b}=4+i 2 \delta,
$$

where the dissipation parameter $\delta \in[0,0.2]$. We focus attention on the region of negative phasevelocity propagation along the $z$ axis with relative wavenumber $\kappa_{i v}$, as illustrated by $w_{i v}<0$ at $0.25<f_{a}<0.42$ in figure 4 .

Real parts of the relative wavenumber $\kappa_{i v}$, calculated at the volume fraction $f_{a}=0.35$ with $\delta=0,0.1$ and 0.2 , are graphed as functions of $\theta$ in figure 7 . The relative wavenumber $\kappa_{i v}$ for the nondissipative FCM (i.e., $\delta=0$ ) is real-valued for $\theta<52^{\circ}$ but has a nonzero imaginary part for $\theta>52^{\circ}$. The relative wavenumbers $\kappa_{i v}$ for $\delta=0.1$ and 0.2 have nonzero imaginary parts for all values of $\theta$. Note that the real part of $\kappa_{i v}$ falls to zero at $\theta=\pi / 2$ in the absence of dissipation (i.e., $\delta=0)$.

Plots of the quantity $w=w_{i v}$, corresponding to the relative wavenumber $\kappa_{i v}$ of figure 7 , are provided in figure 8. The negative phase-velocity condition $w_{i v}<0$ is satisfied

(i) for $\theta<52^{\circ}$ when $\delta=0$,

(ii) for $\theta<76^{\circ}$ when $\delta=0.1$, and

(iii) for $\theta<38^{\circ}$ when $\delta=0.2$.

\section{Discussion and Conclusion}

In isotropic dielectric-magnetic mediums, plane waves can propagate with phase velocity directed opposite to the direction of power flow under certain, rather restrictive, conditions [9]. However, the constitutive parameter space associated with anisotropic and bianisotropic mediums provides a wealth of opportunities for observing and exploiting negative phase-velocity behavior. General conditions are established here for the phase velocity to be directed opposite to power flow for a particular class of bianisotropic mediums, namely Faraday chiral mediums. The theory has been explored by means of a representative example of FCMs, arising from the homogenization of an isotropic chiral medium and a magnetically biased ferrite. For our representative example, the negative phase-velocity conditions have been found to hold for propagation in arbitrary directions - for both nondissipative and dissipative FCMs — provided that the gyrotropic parameter of the ferrite component medium is sufficiently large compared with the corresponding nongyrotropic permeability parameters.

Previous studies [1]-[13] have emphasized the importance of the signs of constitutive (scalar) parameters in establishing the conditions for negative phase-velocity propagation in homogeneous 
mediums. $^{2}$ In the absence of dissipation, negative phase-velocity propagation has been predicted in

(i) isotropic dielectric-magnetic mediums provided that both the permittivity and permeability scalars are negative [3], and

(ii) uniaxial dielectric--magnetic mediums when only one of the four constitutive scalars is negative [10].

Also, the conditions for negative phase-velocity propagation may be fulfilled by dissipative isotropic dielectric-magnetic mediums when only one of the two constitutive scalars has a negative real part [9]. The present study demonstrates that the condition for negative phase-velocity propagation can be satisfied by nondissipative FCMs with constitutive scalars that are all positive. Furthermore, these conditions continue to be satisfied after the introduction a small amount of dissipation.

For the particular case of propagation parallel to the ferrite biasing field, the components of the time-averaged Poynting vector are null-valued in directions perpendicular to the propagation direction. In contrast, for general propagation directions, the time-averaged Poynting vector has nonzero components perpendicular to the direction of propagation. Further studies are required to explore the consequences of the negative phase-velocity condition $\tilde{k}_{R} \underline{\hat{u}} \cdot \underline{P}(\underline{r})<0$ for such general propagation directions.

To conclude, more general bianisotropic mediums, particularly those developed as HCMs based on nonspherical particulate components, offer exciting prospects for future studies of negative phase-velocity propagation.

\section{References}

[1] R.W. Ziolkowski and E. Heyman, Phys. Rev. E 64, 056625 (2001).

[2] A. Lakhtakia, M.W. McCall, and W.S. Weiglhofer, Arch. Elektr. Übertrag. 56, 407 (2002).

[3] V.G. Veselago, in Advances in Electromagnetics of Complex Media and Metamaterials, edited by S. Zouhdi, A. Sihvola and M. Arsalane (Kluwer, Dordrecht, The Netherlands, 2003), p.83

[4] V.G. Veselago, Sov. Phys. Usp. 10, 509 (1968).

[5] D.R. Smith et al., Phys. Rev. Lett. 84, 4184 (2000).

[6] R.A. Shelby, D.R. Smith, and S. Schultz, Science 292, 77 (2001).

[7] J.B. Pendry et al., J. Phys.: Condens. Matter 10, 4785 (1998).

[8] J.B. Pendry et al,, IEEE Trans. Microwave Theory Tech. 47, 2075 (1999).

[9] M.W. McCall, A. Lakhtakia, and W.S. Weiglhofer, Eur. J. Phys. 23, 353 (2002).

\footnotetext{
${ }^{2}$ Parenthetically, negative refraction is also displayed by certain purely dielectric mediums, but they must be nonhomogeneous $[30,31]$.
} 
[10] I.V. Lindell et al., Microw. Opt. Technol. Lett. 31, 129 (2001).

[11] P.M. Valanju, R.M. Walser, and A.P. Valanju, Phys. Rev. Lett. 88, 187401 (2002).

[12] A. Lakhtakia, M.W. McCall, and W.S. Weiglhofer, in Introduction to Complex Mediums for Optics and Electromagnetics, edited by W.S. Weiglhofer and A. Lakhtakia (SPIE Optical Engineering Press, Bellingham, WA, in press).

[13] L. Hu and Z. Lin, Physics Letters A 313, 316 (2003).

[14] M.K. Kärkkäinen, Phys. Rev. E 68, 026602 (2003).

[15] E. Engheta, D.L. Jaggard, and M.W. Kowarz, IEEE Trans. Antennas Propagat. 40, 367 (1992).

[16] W.S. Weiglhofer and A. Lakhtakia, Microw. Opt. Technol. Lett. 17, 405 (1998).

[17] A. Lakhtakia, Beltrami Fields in Chiral Media, (World Scientific, Singapore, 1994).

[18] B. Lax and K.J. Button, Microwave Ferrites and Ferrimagnetics, (McGraw-Hill, New York, NY, 1962).

[19] H.C. Chen, Theory of Electromagnetic Waves, (McGraw-Hill, New York, NY, 1983).

[20] R.E. Collin, Foundations for Microwave Engineering, (McGraw-Hill, New York, NY, 1966), Chap. 6

[21] W.S. Weiglhofer, A. Lakhtakia, and B. Michel, Microwave Opt. Technol. Lett. 18, 342 (1998).

[22] W.S. Weiglhofer and T.G. Mackay, Arch. Elektr. Übertrag. 54, 259 (2000).

[23] A. Lakhtakia (ed), Selected Papers on Linear Optical Composite Materials, (SPIE Optical Engineering Press, Bellingham, WA, 1996).

[24] T.G. Mackay, in Introduction to Complex Mediums for Optics and Electromagnetics, edited by W.S. Weiglhofer and A. Lakhtakia (SPIE Optical Engineering Press, Bellingham, WA, USA, in press).

[25] Z. Fu, H. Zhou, and K. Zhang, Int. J. Infrared Millim. Waves 24, 239 (2003).

[26] T.G. Mackay, A. Lakhtakia, and W.S. Weiglhofer, Arch. Elektr. Übertrag. 55, 243 (2001).

[27] M. Abramowitz and I.A. Stegun (eds.), Handbook of Mathematical Functions, (Dover, New York, NY, 1965).

[28] L. Ward, The Optical Constants of Bulk Materials and Films, (Adam Hilger, Bristol, UK, 1988).

[29] B. Michel et al., Compos. Sci. Technol. 61, 13 (2001).

[30] M. Notomi, Opt. Quantum Electron. 34, 133 (2002).

[31] C. Luo et al., Phys. Rev. B 65, 201104 (2002). 

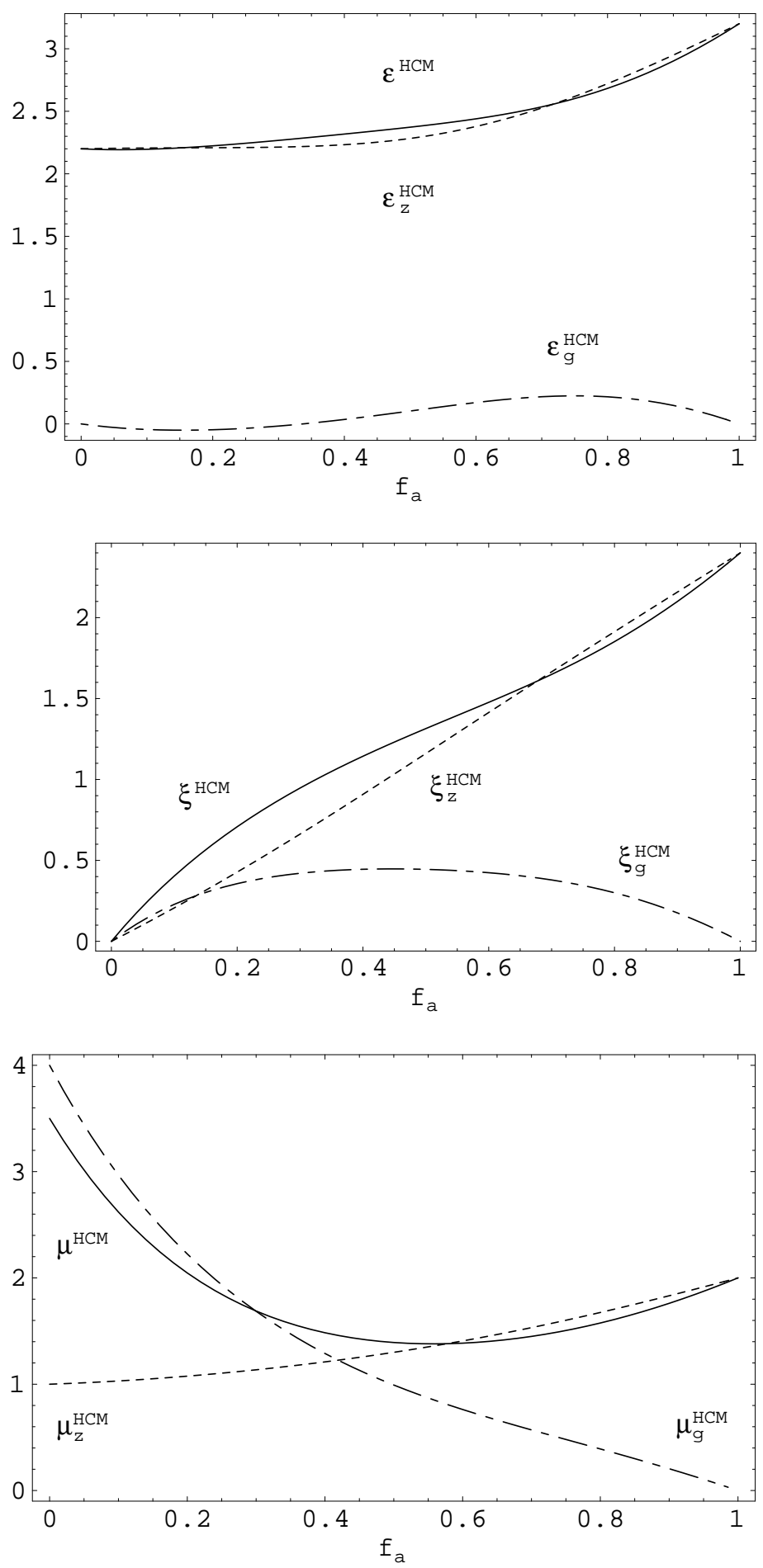

Figure 1: Bruggeman estimates of $\underline{\underline{\epsilon}}^{H C M}, \underline{\underline{\xi}}^{H C M}$ and $\underline{\underline{\mu}}^{H C M}$ as functions of $f_{a}$, when $\epsilon^{a}=3.2$, $\xi^{a}=2.4, \mu^{a}=2, \epsilon^{b}=2.2, \mu^{b}=3.5, \mu_{z}^{b}=\overline{1}$, and $\mu_{g}^{b}=\overline{\overline{4}}$. 

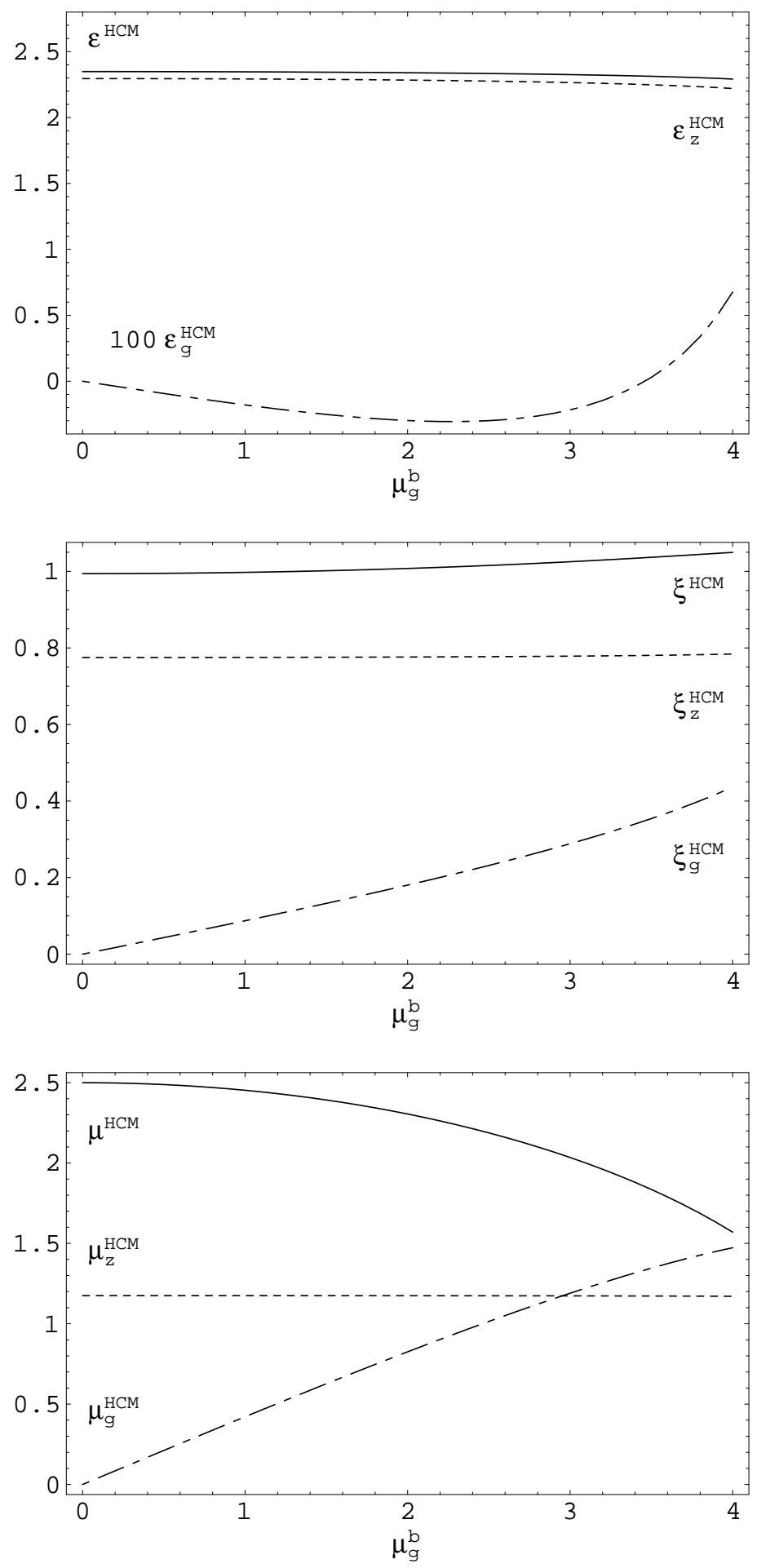

Figure 2: Bruggeman estimates of $\underline{\underline{\epsilon}}^{H C M}, \underline{\underline{\xi}}^{H C M}$ and $\underline{\underline{\mu}}^{H C M}$ as functions of $\mu_{g}^{b}$. The constitutive parameters of the component mediums are the same as in figure 1 , but with $\mu_{g}^{b} \in[0,4]$ and $f_{a}=0.35$. 

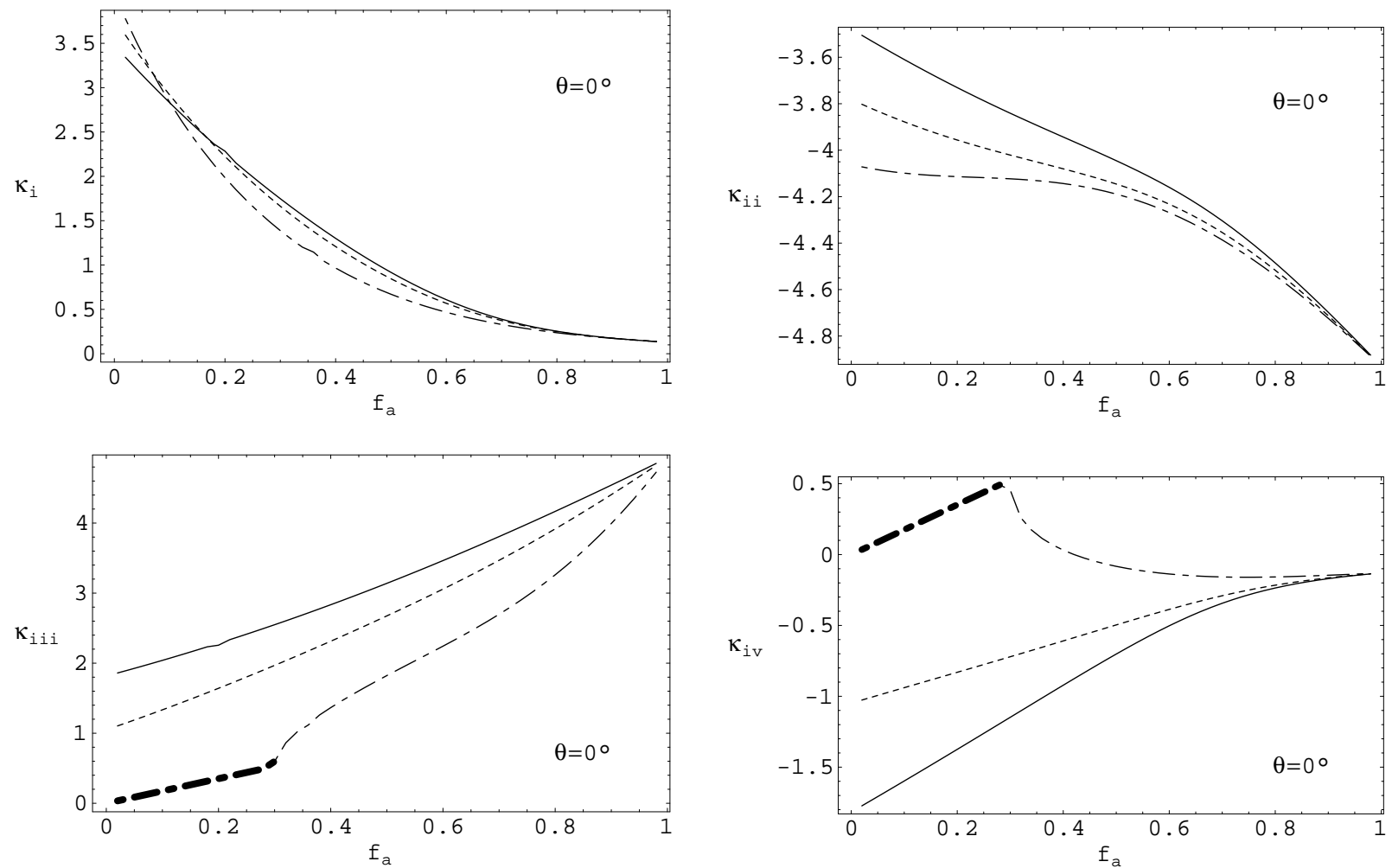

Figure 3: Calculated values of the relative wavenumbers $\kappa_{i-i v}$ as functions of $f_{a}$, when $\theta=0$ and $\mu_{g}^{b}=2,3$ and 4 . The constitutive parameters of the component mediums are: $\epsilon^{a}=3.2, \xi^{a}=2.4$, $\mu^{a}=2, \epsilon^{b}=2.2, \mu^{b}=3.5$, and $\mu_{z}^{b}=1$. Key: $\kappa_{i-i v}$ values corresponding to $\mu_{g}^{b}=2,3$ and 4 are represented by the solid lines, dashed lines, and broken dashed lines, respectively. Heavy lines indicate those relative wavenumbers which have nonzero imaginary parts; the real parts of such complex-valued relative wavenumbers are plotted. 

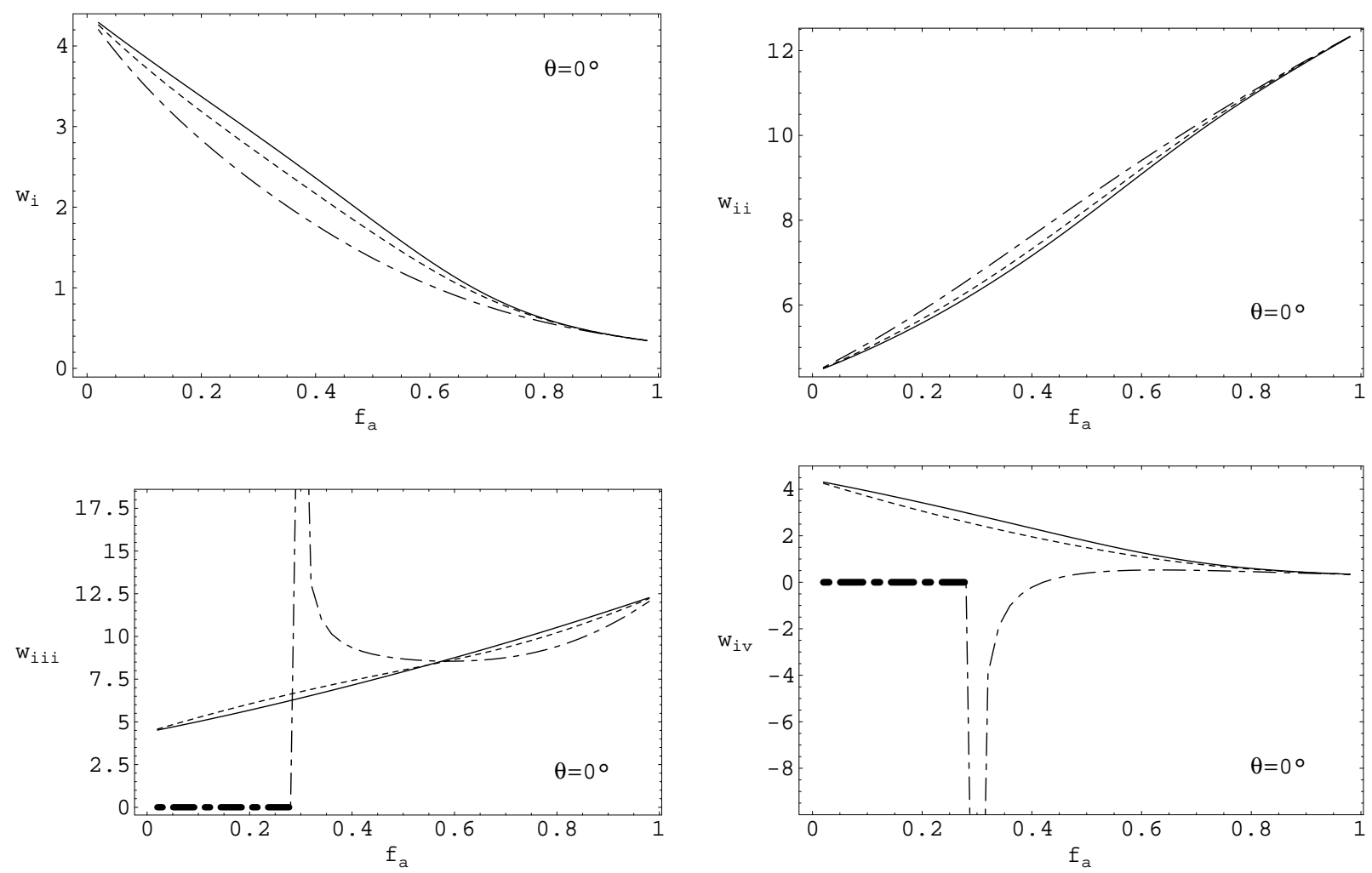

Figure 4: Calculated values of $w_{i-i v}$ as functions of $f_{a}$, when $\theta=0$ and $\mu_{g}^{b}=2,3$ and 4 . The constitutive parameters of the component mediums are the same as in figure 3. Key: $w_{i-i v}$ values corresponding to $\mu_{g}^{b}=2,3$ and 4 are represented by the solid lines, dashed lines, and broken dashed lines, respectively. Heavy lines indicate those $w$ values that devolve from relative wavenumbers $\tilde{k}$ with nonzero imaginary parts. 

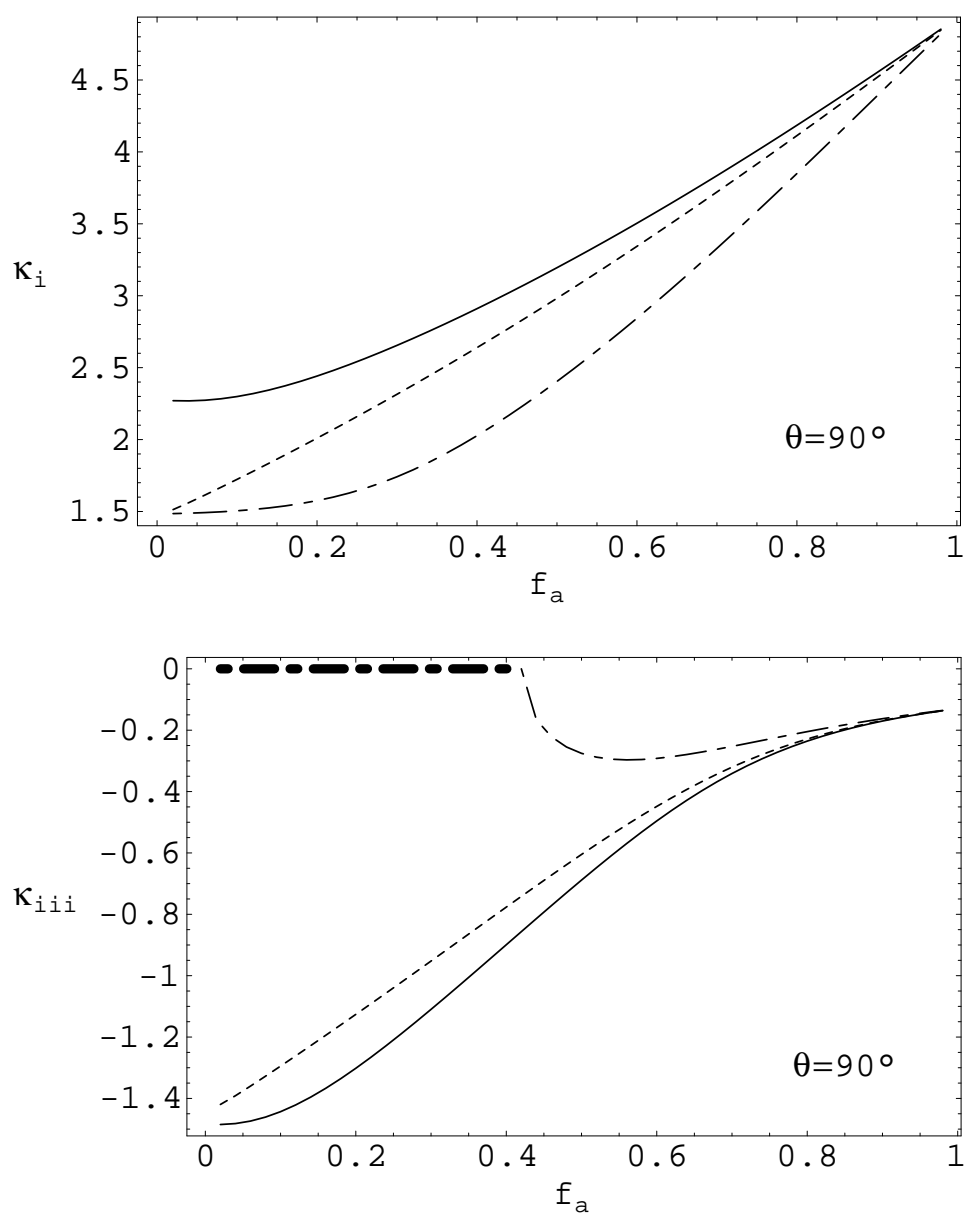

Figure 5: Same as figure 3, but for relative wavenumbers $\kappa_{i, i i i}$ when $\theta=\pi / 2$. 

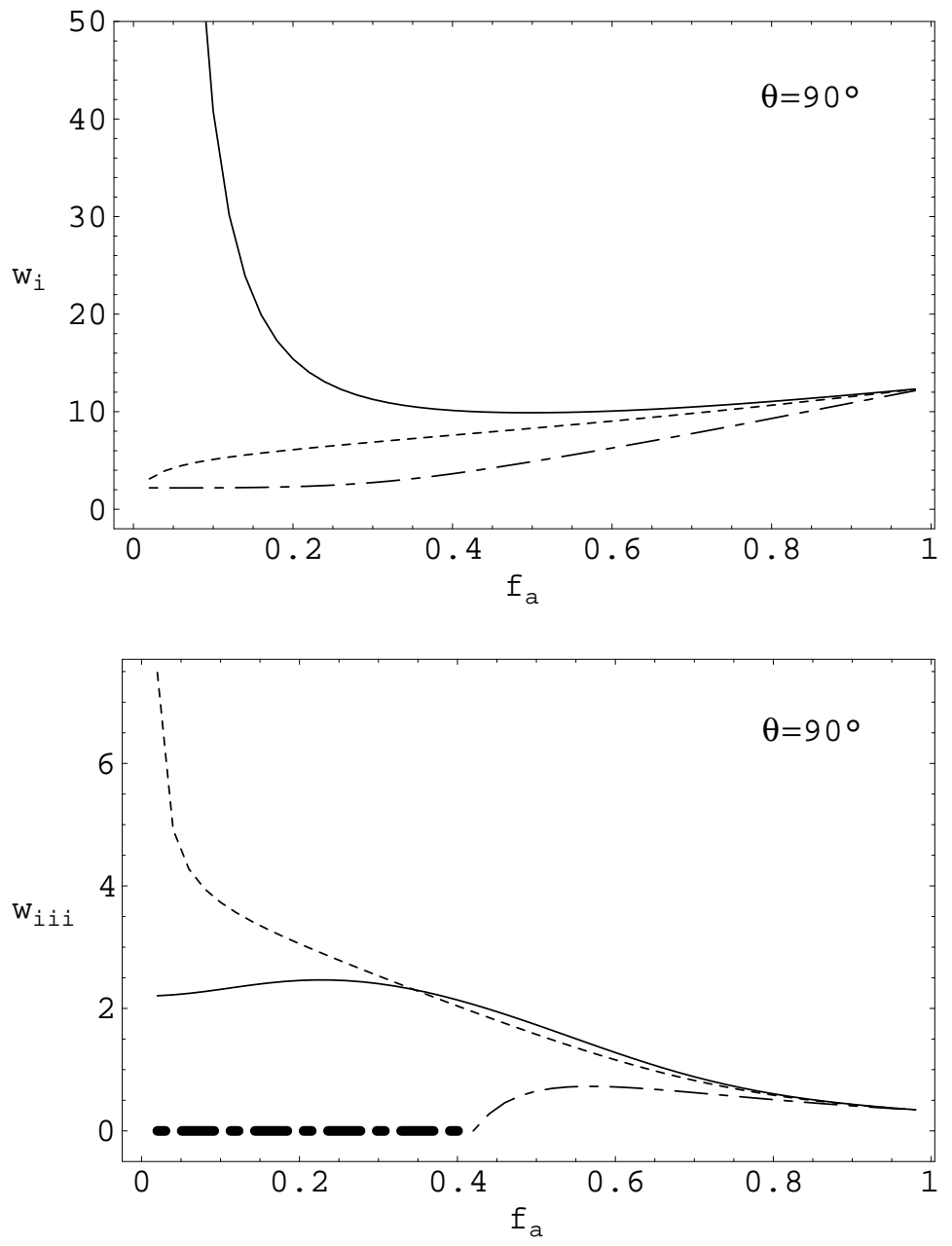

Figure 6: Same as figure 4, but for $w_{i, i i i}$ when $\theta=\pi / 2$. 


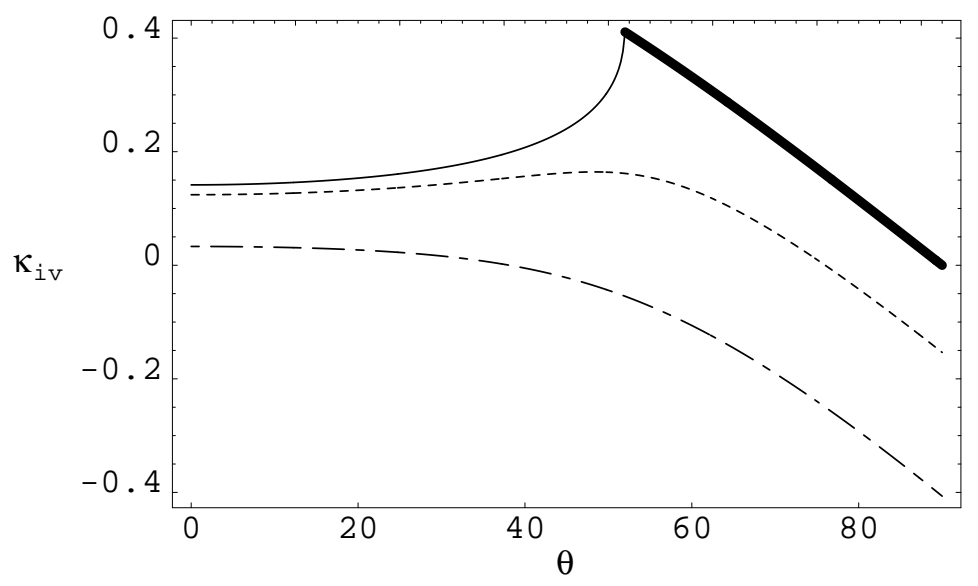

Figure 7: Real parts of relative wavenumbers $\kappa_{i v}$ as functions of $\theta$ (in degrees) for the dissipation parameter $\delta=0,0.1$ and 0.2 when $f_{a}=0.35$. The constitutive parameters of the component mediums are: $\epsilon^{a}=3.2, \xi^{a}=2.4, \mu^{a}=2, \epsilon^{b}=2.2+i \delta, \mu^{b}=3.5+i \delta, \mu_{z}^{b}=1+i 0.5 \delta$, and $\mu_{g}^{b}=4+i 2 \delta$. Key: $\kappa_{i v}$ values corresponding to $\delta=0,0.1$ and 0.2 are represented by the solid lines, dashed lines, and broken dashed lines, respectively. The heavy line on the graph for $\delta=0$ indicates those relative wavenumbers $\kappa_{i v}$ which have nonzero imaginary parts.

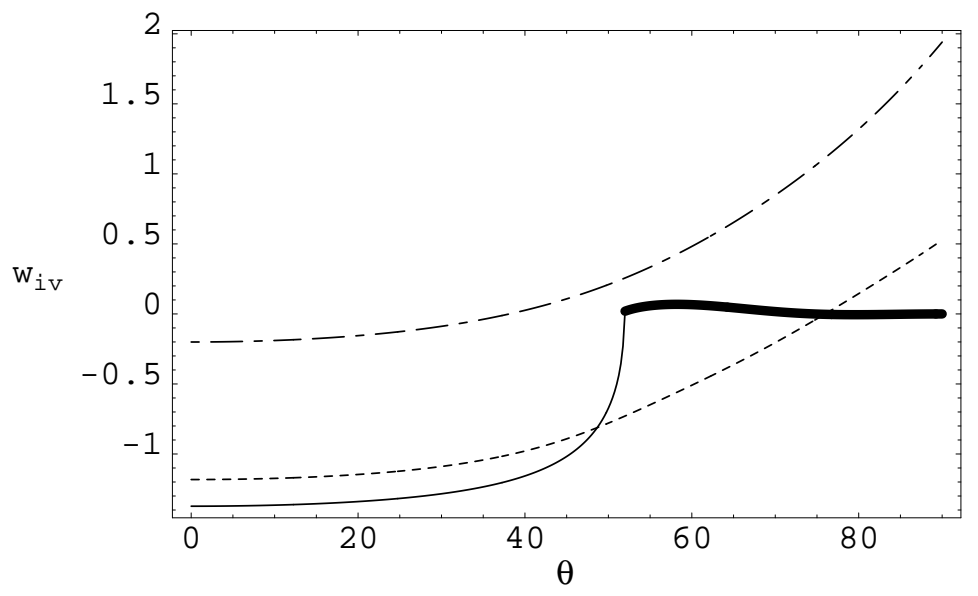

Figure 8: Calculated values of $w_{i v}$ as functions of $\theta$ (in degrees) for the dissipation parameter $\delta=0,0.1$ and 0.2 when $f_{a}=0.35$. The constitutive parameters of the component mediums are as in figure 7. Key: $w_{i v}$ values corresponding to $\delta=0,0.1$ and 0.2 are represented by the solid lines, dashed lines, and broken dashed lines, respectively. The heavy line on the graph for $\delta=0$ indicates those $w_{i v}$ values which devolve from relative wavenumbers $\kappa_{i v}$ with nonzero imaginary parts. 